\title{
Abnormal mGlu 5 Receptor/Endocannabinoid Coupling in Mice Lacking FMRP and $\mathrm{BCI}$ RNA
}

\author{
Mauro Maccarrone*,1,2, Silvia Rossi ${ }^{2,3}$, Monica Bari, ${ }^{2,4}$, Valentina De Chiara ${ }^{2,3}$, Cinzia Rapino ${ }^{1,2}$, \\ Alessandra Musella ${ }^{2,3}$, Giorgio Bernardi ${ }^{2,3}$, Claudia Bagni ${ }^{5,6}$ and Diego Centonze ${ }^{*, 2,3}$ \\ 'Dipartimento di Scienze Biomediche, Università degli Studi di Teramo, Teramo, Italy; ${ }^{2}$ Fondazione Santa Lucia, Rome, Italy; ${ }^{3}$ Clinica Neurologica, \\ Dipartimento di Neuroscienze, Università Tor Vergata, Rome, Italy; ${ }^{4}$ Dipartimento di Medicina Sperimentale e Scienze Biochimiche, Università Tor \\ Vergata, Rome, Italy; ${ }^{5}$ Center for Human Genetics, Katholieke Universiteit Leuven, Leuven, Belgium; ${ }^{6}$ Department of Molecular and \\ Developmental Genetics, Flanders Institute for Biotechnology (VIB), Leuven, Belgium
}

Transcriptional silencing of the gene encoding the fragile $X$ mental retardation protein (FMRP) causes fragile $X$ syndrome (FXS). FMRP acts as a translational repressor at central synapses, and molecular and synaptic plasticity studies have shown that the absence of this protein alters metabotropic glutamate 5 receptors (mGlu5Rs)-mediated signaling. In the striatum of mice lacking FMRP, we found enhanced activity of diacylglycerol lipase (DAGL), the enzyme limiting 2-arachidonoylglicerol (2-AG) synthesis, associated with altered sensitivity of GABA synapses to the mobilization of this endocannabinoid by mGlu5R stimulation with DHPG. Mice lacking another repressor of synaptic protein synthesis, BCI RNA, also showed potentiated mGlu5R-driven 2-AG responses, indicating that both FMRP and $\mathrm{BCI}$ RNA act as physiological constraints of mGlu5R/endocannabinoid coupling at central synapses. The effects of FMRP ablation on DAGL activity and on DHPG-mediated inhibition of GABA synapses were enhanced by simultaneous genetic inactivation of FMRP and $\mathrm{BCI}$ RNA. In double FMRP and BCI RNA lacking mice, striatal levels of 2-AG were also enhanced compared with control animals and to single mutants. Our data indicate for the first time that $\mathrm{mGlu5R}$-driven endocannabinoid signaling in the striatum is under the control of both FMRP and BCI RNA. The abnormal mGlu5R/2-AG coupling found in FMRP-KO mice emphasizes the involvement of mGlu5Rs in the synaptic defects of FXS, and identifies the modulation of the endocannabinoid system as a novel target for the treatment of this severe neuropsychiatric disorder.

Neuropsychopharmacology (2010) 35, 1500-1509; doi:I0.1038/npp.2010.19; published online 10 March 2010

Keywords: 2-AG; anandamide; DHPG; IPSC; fragile X; synaptic plasticity

\section{INTRODUCTION}

Fragile X syndrome (FXS) is a familial form of mental retardation, and the predominant monogenic cause of autism. It is due to mutation of the fragile $\mathrm{X}$ mental retardation $(f m r 1)$ gene, resulting in its transcriptional silencing and absence of the corresponding protein product, the fragile $\mathrm{X}$ mental retardation protein (FMRP) (Bassell and Warren, 2008). A primary role of FMRP in the brain is to control synaptic transmission and plasticity, by regulating mRNA metabolism and repressing protein synthesis in dendrites and synapses (Bagni and Greenough, 2005; Sutton

*Correspondence: Professor D Centonze, Dipartimento di Neuroscienze, Università Tor Vergata, Via Montpellier I, Rome 00133 , Italy, Tel: + 390672596010 , Fax: + 39067259 6006, E-mail: centonze@uniroma2.it

or M Maccarrone, Dipartimento di Scienze Biomediche, Università degli Studi di Teramo, Piazza A. Moro 45, Teramo 64100, Italy, Tel: + $39086 \mid$ 266875; Fax: + 39 086I 266877, E-mail: mmaccarrone@unite.it

Received 13 October 2009; revised 13 January 2010; accepted 3 February 2010 and Schuman, 2006; Schuman et al, 2006). The lack of this protein leads to excessive protein synthesis, and causes a complex neuropsychiatric and somatic phenotype, which is replicated to a certain extent in the animal model of FXS, the FMRP-knockout (FMRP-KO) mouse (Dölen et al, 2007; Bassell and Warren, 2008).

Local translation of mRNAs at synapses is also regulated by a variety of small non-protein-coding RNAs, important components of the human transcriptome recently recognized to have an instrumental role in translational control (Zalfa et al, 2006). The Brain cytoplasmic 1 RNA (BC1 RNA) is a regulatory RNA which cooperates with FMRP in the control of protein synthesis at synapses (Zalfa et al, 2003), and acts, in vitro, as a general translational repressor at the initiation level (Wang et al, 2002, 2005). A physical association between FMRP and BC1 RNA in brain and at synapses has been described (Zalfa et al, 2003; Johnson et al, 2006), as well as a cooperative action of the two regulatory molecules in the control of GABA transmission in the striatum (Centonze et al, 2008).

Recently, BC1 RNA has been proposed to regulate, under synaptic activity, two FMRP target mRNAs: FMR1 and 
PSD-95 (Zhong et al, 2009), further linking FMRP and BC1 in the control of neuronal mRNA translation.

Early studies addressing hippocampal synaptic plasticity in FMR1-KO mice revealed that the activity of metabotropic glutamate 5 receptors (mGlu5Rs) is enhanced in this model of FXS, giving rise to the idea that FMRP and mGlu5Rs work in functional opposition, and that the absence of FMRP leads to exaggerated signaling through mGlu5Rs, ultimately causing FXS. According to this 'mGluR theory' of FXS (Bear et al, 2004), genetic reduction of mGlu5R signaling can reverse the neurological defects typical of FXS in FMRP-KO mice (Dölen et al, 2007; Dölen and Bear, 2008). More recently, enhanced mGlu5R-dependent neuronal excitability has been reported in mice lacking BC1 RNA (BC1-KO mice) (Zhong et al, 2009), and this finding might indicate that FMRP and BC1 RNA interact functionally to control the activity of these receptors.

The stimulation of mGlu5Rs activates a complex cascade of intracellular events in dendrites, and leads to the mobilization of the endocannabinoid system. Accordingly, pharmacological activation of mGlu5Rs has been repeatedly shown to increase the levels of the endocannabinoid 2-arachidonoylglicerol (2-AG) (Jung et al, 2005; Maccarrone et al, 2008), and to inhibit transmitter release through presynaptic cannabinoid $\mathrm{CB} 1$ receptors (CB1Rs) (Maccarrone et al, 2008). Based on the notion that mGlu5Rs are dysregulated in FMRP-KO and in BC1-KO mice, it can be postulated that mGlu5R-stimulated 2-AG metabolism and physiological activity are also altered in these mutants. We addressed this hypothesis by measuring mGlu5R/2-AG coupling in the striatum of FMRP-KO mice, and comparing the effects with those obtained in BC1-KO mice, and in double mouse mutants lacking both FMRP and $\mathrm{BC} 1 \mathrm{RNA}$ (FMRP-BC1-KO mice).

\section{MATERIALS AND METHODS}

Mice lacking fmr1 (FMRP-KO), BC1 RNA (BC1-KO), and both fmr1 and BC1 RNA (FMRP-BC1-KO) were employed along with their respective, age-matched wild-type (WT) strains (2-3 months old), as already reported (Centonze et al, 2007a, 2008). Corticostriatal coronal slices $(200 \mu \mathrm{m})$ were prepared from brain blocks with the use of a vibratome (Maccarrone et al, 2008). All efforts were made to minimize animal suffering and to reduce the number of mice used in accordance with the European Communities Council Directive of 24 November, 1986 (86/609/EEC).

\section{Electrophysiology}

Single slices were transferred to a recording chamber and submerged in a continuously flowing artificial cerebrospinal fluid (ACSF) $\left(33^{\circ} \mathrm{C}, 2-3 \mathrm{ml} \mathrm{min}^{-1}\right)$ gassed with $95 \% \mathrm{O}_{2}-5 \%$ $\mathrm{CO}_{2}$. The composition of the bathing solution was (in $\mathrm{mM}$ ): (126) $\mathrm{NaCl}$, (2.5) $\mathrm{KCl}$, (1.2) $\mathrm{MgCl}_{2}$, (1.2) $\mathrm{NaH}_{2} \mathrm{PO}_{4}$, (2.4) $\mathrm{CaCl}_{2}$, (11) Glucose, (25) $\mathrm{NaHCO}_{3}$.

Recordings were made with borosilicate glass pipettes (1.8 mm, 2-4 M $\Omega$ ) by using an Axopatch 1D patch clamp amplifier (Molecular Devices, Foster City, CA, USA) under visual inspection (Centonze et al, 2007a, 2008; Maccarrone et al, 2008). Whole-cell access resistances were in the range of 5-20 M $\Omega$.

To study GABA transmission, recording pipettes were filled with internal solution of the following composition $(\mathrm{mM}): \mathrm{CsCl}(110), \mathrm{K}^{+}$-gluconate (30), ethylene glycol-bis ( $\beta$-aminoethyl ether)- $N, N, N^{\prime}, N^{\prime}$-tetra-acetic acid (EGTA; 1.1), HEPES (10), $\mathrm{CaCl}_{2}$ (0.1), Mg-ATP (4), Na-GTP (0.3). MK-801 and CNQX were added to the external solution to block, NMDA and nonNMDA glutamate receptors, respectively. The identification of putative spiny neurons was achieved immediately after rupture of the $G \Omega$ seal by evaluating the firing response to the injecting of depolarizing current and/or pharmacologically since DHPG does not alter the membrane properties of these cells, while causing membrane excitation in interneurons (Centonze et al, 2007b; Bonsi et al, 2008).

To study glutamate transmission, the recording pipettes were filled with internal solution of the following composition $(\mathrm{mM}): \mathrm{K}^{+}$-gluconate (125), $\mathrm{NaCl}(10), \mathrm{CaCl}_{2},(1.0)$, $\mathrm{MgCl}_{2}$ (2.0), 1,2-bis (2-aminophenoxy) ethane- $N, N, N, N$ tetra-acetic acid (BAPTA; 0.5), HEPES (19), guanosine triphosphate (GTP; 0.3), Mg-adenosine triphosphate (Mg-ATP; 1.0), adjusted to $\mathrm{pH} 7.3$ with $\mathrm{KOH}$. Bicuculline was added to the perfusing solution to block GABAAmediated transmission.

All synaptic events were recorded at the holding potential of $-80 \mathrm{mV}$ and stored by using P-CLAMP 9 (Molecular Devices, Foster City, CA, USA).

Miniature and spontaneous synaptic currents (mIPSCs, sIPSCs, mEPSCs) were analyzed off line as described (Centonze et al, 2007a, 2008; Maccarrone et al, 2008). DHPG was applied for $12 \mathrm{~min}$, and statistical significance was calculated by comparing the pre-drug value with that recorded immediately before DHPG wash out.

Drugs were applied by dissolving them to the desired final concentration in the bathing ACSF. The concentrations of the drugs were as follows: AM251 $(10 \mu \mathrm{M})$, CNQX $(10 \mu \mathrm{M})$, 3,5-DHPG (DHPG, $50 \mu \mathrm{M})$, HU210 $(1 \mu \mathrm{M})$, MK-801 $(30 \mu \mathrm{M})$, MPEP $(30 \mu \mathrm{M})$, pirenzepine dihydrochloride (pirenzepine, $100 \mathrm{nM}$ ), tetrodotoxin (TTX, $1 \mu \mathrm{M}$ ), (from Tocris Cookson, Bristol, UK). Bicuculline $(10 \mu \mathrm{M})$, McN-A-343 $(3 \mu \mathrm{M})$, orlistat $(5 \mu \mathrm{M})$ (from Sigma-RBI, St Louis, MO, USA).

\section{Biochemistry}

Intact slices were subjected to biochemical measurements after tissue processing. The activity of diacylglycerol lipase (DAGL) was assayed with $10 \mu \mathrm{M} s n$-1-stearoyl-2- $\left[{ }^{14} \mathrm{C}\right]$ arachidonoyl-glycerol (Perkin Elmer Life Sciences, Boston, MA, USA) as substrate, and that of monoacylglycerol lipase (MAGL) was determined using $10 \mu \mathrm{M}$ 2-oleoyl- $\left[{ }^{3} \mathrm{H}\right]$-glycerol (ARC, St Louis, MO, USA) as substrate (Maccarrone et al, 2008). Both DAGL and MAGL activities were expressed as pmol product $\mathrm{min}^{-1} \mathrm{mg}^{-1}$ protein.

For the evaluation of endogenous levels of 2-AG, striatal slices were subjected to lipid extraction with chloroform/ methanol $(2: 1, \mathrm{v} / \mathrm{v})$, in the presence of deuterated 2-AG (d8-2-AG, from Cayman Chemicals (Ann Arbor, MI, USA) as internal standard (Giuffrida et al, 2000). The organic phase was dried and then analyzed by liquid chromatography-electrospray ionization mass spectrometry (LC-ESIMS), using a single quadrupole API-150X mass spectrometer 
(Applied Biosystem, Foster city, CA, USA) coupled with a Perkin Elmer LC system (Perkin Elmer, Waltham, MA, USA). Quantitative analysis was performed by selected ion recording over the respective sodiated molecular ions (Giuffrida et al, 2000).

For mGlu5R studies, mouse striatal slices were homogenized in ice-cold assay buffer, consisting of $50 \mathrm{mM}$ Tris- $\mathrm{HCl}$ ( $\mathrm{pH} 7.5$ ), 0.9\% (wt/vol) $\mathrm{NaCl}$ (Maccarrone et al, 2009). The homogenate was centrifuged at $39000 \times g$ for $10 \mathrm{~min}$ at $4{ }^{\circ} \mathrm{C}$, and the pellet was resuspended in assay buffer immediately before use. Samples $(100 \mu \mathrm{g}$ protein/test $)$ were incubated with $1 \mathrm{nM}\left[{ }^{3} \mathrm{H}\right] \mathrm{MPEP}$ in a $550 \mu \mathrm{l}$ reaction buffer, and non-specific binding was determined in the presence of a $10 \mu \mathrm{M}$ excess of 'cold' MPEP (Maccarrone et al, 2009). After incubation for $1 \mathrm{~h}$ at $30^{\circ} \mathrm{C}$, samples were subjected to rapid filtration assays. Filter radioactivity was quantified by liquid scintillation counting. In preliminary experiments saturation curves were drawn to determine apparent dissociation constant $(\mathrm{Kd})$ and maximum binding (Bmax) values for WT and transgenic mice. From these curves, it was found that all strains had a similar $\mathrm{Kd}$ $(\sim 1.0 \mathrm{nM})$ and different Bmax values $(23.4 \pm 0.9 ; 25.7 \pm 1.3$; $77.1 \pm 5.6 ; 64.8 \pm 5.3 \mathrm{fmol} / \mathrm{mg}$ protein for $\mathrm{WT}, \mathrm{BC} 1-\mathrm{KO}$, FMRP-KO, and FMRP-BC1-KO mice, respectively). On this basis, binding assays were performed at $1.0 \mathrm{nM}\left[{ }^{3}\right] \mathrm{MPEP}$, as already reported (Domenici et al, 2005).

\section{Statistical Analysis}

Electrophysiological data are presented as means \pm SEM. Statistical analysis was performed using a paired or unpaired Student's $t$-test or Wilcoxon's test. Biochemical data are presented as means $\pm S D$ of at least three independent experiments, each performed in duplicate. Statistical analysis was performed by the nonparametric Mann-Whitney $U$-test, elaborating experimental data by means of the InStat 3 program (GraphPAD Software for Science, San Diego, CA). The significance level was established at $p<0.05$.

\section{RESULTS}

\section{Effects of DHPG on Striatal mIPSCs in FMRP-KO Mice}

As previously reported (Maccarrone et al, 2008, 2009), application of the group I mGlu receptor agonist DHPG inhibited mIPSC frequency in striatal neurons recorded from WT mice $(n=18 / 22$ cells, $p<0.01)$ (Figure $1 \mathrm{a})$. This effect was associated with negligible changes of mIPSC amplitude $(p>0.05)$ (not shown), and was secondary to the stimulation of CB1Rs by endocannabinoids released in response to mGlu5R activation. Accordingly, the DHPGmediated inhibition of mIPSC frequency was fully prevented by preincubation (10 min) with AM251 (a cannabinoid CB1R antagonist), or by MPEP (a mGlu5R antagonist) ( $n=5 / 5$ and $p>0.05$ for the two experimental conditions) (Figure 1b). As in WT animals, the majority of striatal neurons from FMRP-KO mice was sensitive to the effects of DHPG $(n=16 / 27)$, but the effect of this agonist on mIPSC frequency was remarkably potentiated in responsive neurons $(p<0.01$ compared with pre-DHPG values in FMRP-KOs, and $p<0.05$ compared with DHPG response of WT neurons) (Figure $1 \mathrm{a}, \mathrm{c}$ and $\mathrm{d}$ ). In the presence of AM251 or of MPEP, DHPG failed to change MIPSC frequency in all the recorded FMRP-KO neurons $(n=10$ / 10 and $p>0.05$ for the two groups; Figure $1 \mathrm{~b}$ ).

Taken together, these data indicate that FMRP ablation causes a profound perturbation of the interaction between mGlu5Rs and the endocannabinoid system in the striatum.

\section{Striatal mGlu5R Expression and Function in FMRP-KO Mice}

FMRP regulates mGlu5R activity (Dölen and Bear, 2008). Thus, we first explored MPEP binding in both WT and FMRP-KO mice, and found that ablation of $f m r l$ gene caused a dramatic elevation of mGlu5R expression in the striatum $(n=4, p<0.001)$ (Figure $2 \mathrm{a})$. This alteration was associated with a parallel increase of the activity of DAGL, which is the enzyme responsible for the synthesis of the endocannabinoid 2-AG (Bisogno et al, 2003), either in the absence or in the presence of DHPG $(n=4$ and $p<0.01$ for both conditions) (Figure $2 \mathrm{a}$ ). This observation is consistent with previous findings showing that mGlu5Rs stimulate 2-AG release and physiological activity by enhancing DAGL activity in the striatum (Maccarrone et al, 2008). To further confirm that DAGL-stimulated 2-AG synthesis was involved in the physiological activity of mGlu5R stimulation, we preincubated striatal slices with the lipase inhibitor orlistat before the application of DHPG. In the presence of orlistat, that blocks DAGL at low nM concentrations (Bisogno et al, 2006), and hence blunts DAGL-dependent events (Hoover et al, 2008), DHPG failed to inhibit mIPSC frequency $(p>0.05)$ in both WT $(n=6 / 6)$ and FMRP-KO mice $(n=8 / 9)$ (Figure 2b).

Some actions of DHPG on striatal GABA synapses are independent of endocannabinoid mobilization, because they depend on direct depolarization and action potential generation in GABAergic interneurons, which synapse onto striatal projection cells (Centonze et al, 2007b). Thus, to see whether FMRP also controls the 2-AG-independent action of DHPG on striatal GABA transmission, we measured the frequency of sIPSCs in the presence of AM251, to block CB1Rs. Under these experimental conditions, DHPG increased to a similar extent sIPSC frequency in striatal neurons from both WT and FMRP-KO mice $(n=8 / 8$, $p<0.01$ for both groups) (Figure $2 \mathrm{c}$ ).

\section{Effects of the Direct Modulation of Cannabinoid CB1Rs in FMRP-KO Mice}

The increased activity of DAGL in the striatum of FMRP-KO mice might indicate the existence of an endocannabinoid tone in these mutants. We explored this possibility by measuring the effects of CB1R blockade on mIPSC frequency in FMRP-KO mice. AM251 (10 min) failed to increase mIPSC frequency in both WT $(n=9 / 9)$ and FMRPKO neurons $(n=12 / 12 p>0.05$ (Figure $3 \mathrm{a})$. We then measured 2-AG contents following $f m r 1$ gene ablation and, consistently with the electrophysiological data, we found no difference in striatal levels of 2-AG in WT and FMRP-KO mice $(n=4, p>0.05)$ (Figure $3 \mathrm{~b})$. To provide a possible explanation for these results, we also measured the 
a

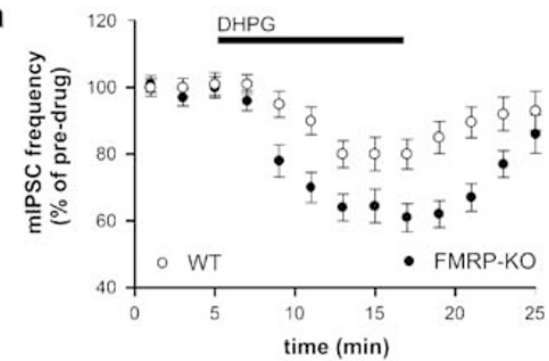

C
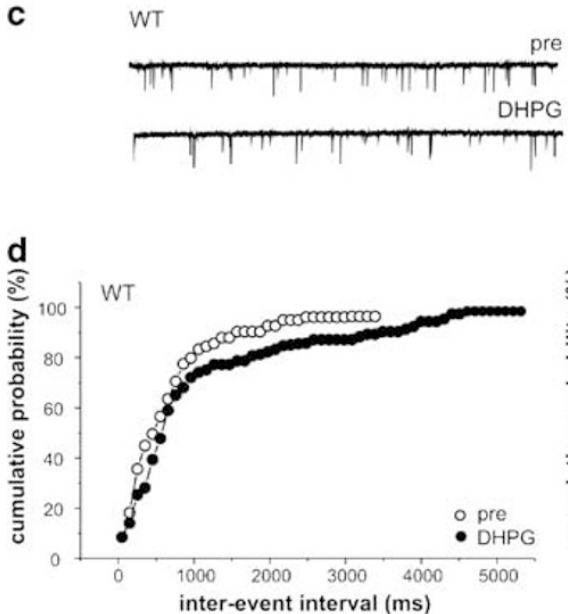

b

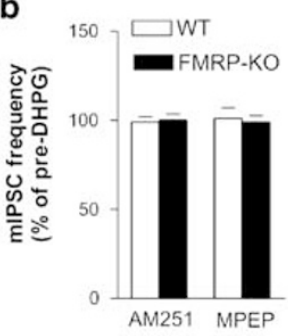

FMRP-KO
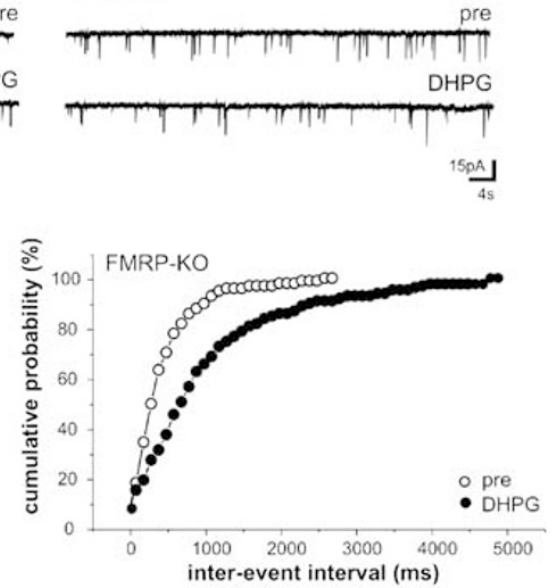

Figure I Effects of DHPG on striatal mIPSCs in FMRP-KO mice. (a) The reduction of mIPSC frequency induced by the group I mGlu receptor agonist DHPG was potentiated in FMRP-KO mice. (b) Preincubation with the CBI receptor antagonist AM25I or with mGlu5 receptor antagonist MPEP prevented the depressant action of DHPG in WT and FMRP-KO mice. (c) Examples of voltage-clamp recordings before and during the application of DHPG in control and FMRP-KO mice. (d) Cumulative distribution of mIPSC inter-event interval recorded from WT and FMRP-KO mice before and during the application of DHPG.

activity of MAGL, the main activity responsible for 2-AG degradation (Dinh et al, 2002). Consistent with the idea that 2-AG degradation is enhanced in FMRP-KO mice, we observed increased activity of MAGL in these mutants $(n=4, p<0.01)$. It is interesting to note that DHPG significantly reduced the activity of MAGL in FMRP-KO mice $(n=4, p<0.01$ compared with pre-DHPG), but 2-AG contents were not increased by this treatment $(n=4$, $p>0.05$ compared with pre-DHPG) (Figure $3 b$ ). This latter finding might reflect the electrophysiological evidence that DHPG seems to stimulate 2-AG synthesis only in a set of GABA synapses in FMRP-KO mice. Alternatively, it is also conceivable that regulatory mechanisms other than DAGL and MAGL, possibly exacerbated in FMRP-KO mice, might be involved in the control of 2-AG metabolism (Di Marzo and Maccarrone, 2008).

We also studied the sensitivity of striatal CB1Rs to a direct pharmacological agonist of these receptors, because the abnormal responses of GABA synapses to mGlu5Rtriggered 2-AG signaling might indicate that $\mathrm{CB} 1 \mathrm{R}$ function is altered in FMRP-KO mice. In these mutants, however, the CB1R agonist HU210 produced similar inhibition of mIPSC frequency than in the respective WT counterparts (FMRPKO: $n=8 / 10$, WT: $n=8 / 9 ; p<0.01$ for both genotypes) (Figure 3c). HU210 also produced comparable inhibition of glutamate-mediated mEPSC frequency in WT $(n=7$, $p<0.01)$ and in FMRP-KO neurons $(n=9, p<0.01)$, indicating that FMRP does not have a major role in the regulation of CB1Rs controlling inhibitory or excitatory synaptic transmission in the striatum (Figure $3 \mathrm{~d}$ ).

\section{Effects of fmrl Gene Ablation on Muscarinic M1 - Endocannabinoid Interaction in the Striatum}

The results described above might indicate that the abnormal responses of GABA synapses to DHPG in FMRP-KO neurons rely on altered 2-AG metabolism rather than on abnormal sensitivity of mGlu5 or CB1Rs to the respective agonists. Activation of muscarinic M1 receptors also mobilizes 2-AG in the striatum, and inhibits GABA synapses through the stimulation of CB1Rs (Narushima et al, 2007). Thus, to see whether the observed alterations of endocannabinoid signaling in FMRP-KO mice were specific for mGlu5Rs or also involved muscarinic $\mathrm{M} 1$ receptors, we measured the effects of McN-A-343, a selective agonist of M1 receptors, on mIPSCs recorded from WT and FMRP-KO mice. McN-A-343 reduced mIPSC frequency in WT mice $(n=8 / 11, p<0.01)$ without altering the amplitude of these events $(p>0.05)$. In FMRP$\mathrm{KO}$ mice, the effects of McN-A-343 were similar to those recorded in their control counterparts $(n=9 / 11, p<0.01)$ (Figure 3e). In both WT and FMRP-KO mice, the effects of McN-A-343 were fully prevented by the M1R antagonist pirenzepine ( $n=6 / 6$ and $p>0.05$ for both groups) (not shown), or by AM251 ( $n=5 / 5$ and $p>0.05$ for both groups), indicating that they were mediated by M1R-stimulated endocannabinoids acting on CB1Rs (Figure 3f).

\section{mGlu5R/Endocannabinoid Interaction in BC1-KO Mice}

Part of the physiological activity of FMRP at synapses is mediated by the interaction of this protein with $\mathrm{BC} 1 \mathrm{RNA}$ 

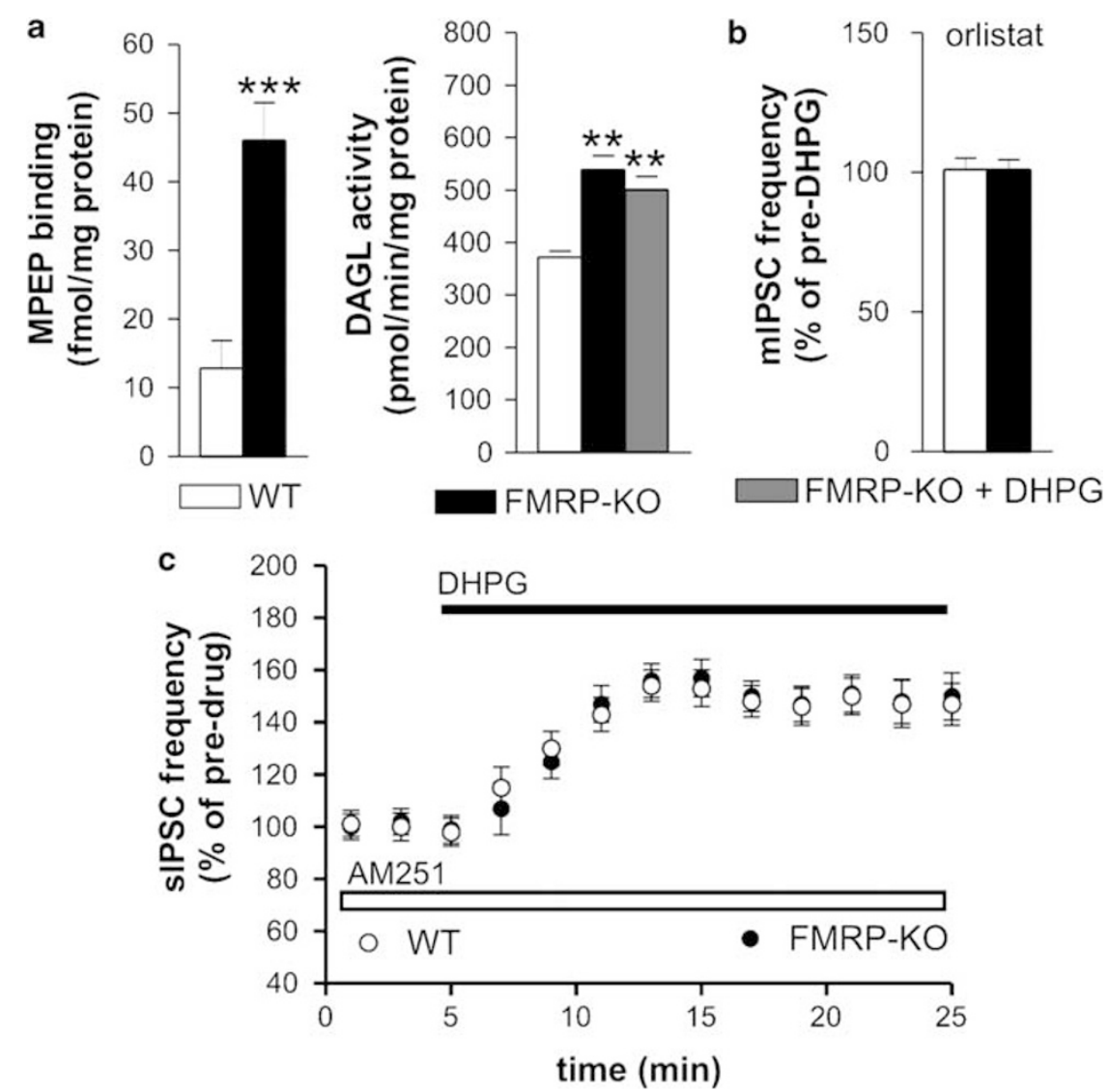

Figure 2 Striatal $m$ Glu5R expression and function in FMRP-KO mice. (a) The genetic ablation of fmrl gene markedly increased MPEP binding to mGlu5R, and DAGL activity in the striatum. (b) Preincubation with the DAGL inhibitor orlistat prevented the depressant action of DHPG on mIPSC frequency in WT and FMRP-KO mice. (c) 2-AG-independent action of DHPG on sIPSC frequency was similar in striatal neurons from both WT and FMRP-KO mice. *** $p<0.01$; **** $p<0.001$.

(Zalfa et al, 2003, Napoli et al, 2008), a small non-coding RNA possibly involved, as FMRP, in translation regulation in dendrites. Thus, we recorded the effects of DHPG in mice lacking $\mathrm{BC} 1 \mathrm{RNA}$, to see whether the altered responses seen in FMRP-KO mice were also present in these mutants. As already described (Centonze et al, 2008), the frequency of mIPSCs was higher in BC1-KO striatal neurons $(2.8 \pm 0.4 \mathrm{~Hz} ; n=18)$ than in controls $(1.4 \pm 0.3 \mathrm{~Hz} ; n=16)$ $(p<0.01)$, and similar to that of FMRP-KOs $(2.6 \pm 0.4 \mathrm{~Hz}$; $n=19)(p>0.05)$ (not shown). Administration of DHPG resulted in a potentiated inhibition of mIPSC frequency in BC1-KO striatal cells $(n=18 / 21)$, reminiscent of the effect seen in FMRP-KO mice $(p<0.001$ compared with preDHPG value, $p<0.01$ compared with DHPG effect in WT mice, $p>0.05$ compared with DHPG effect in FMRP-KOs) (Figure 4a). Also in the mutants, in fact, MPEP $(n=6 / 6)$ and AM251 $(n=6 / 6)$ fully blocked the effect of DHPG $(p>0.05$ for both antagonists) (Figure $4 \mathrm{~b}$ ).

Analysis of the mechanism at the basis of DHPGmediated inhibition of striatal mIPSCs, however, revealed that FMRP and BC1 RNA ablation altered mGlu5/2-AG coupling in remarkably distinct ways. MPEP binding and basal DAGL activity, in fact, were similar in BC1-KO mice and in WT mice $(p>0.05)$, and significantly reduced compared with FMRP-KOs $(p<0.001$ for MPEP binding and $p<0.01$ for DAGL activity) (Figure $4 \mathrm{c}$ ). In the presence of DHPG, however, the activity of DAGL was dramatically enhanced in $\mathrm{BC} 1-\mathrm{KO}$ mice, reaching levels even higher than those seen in WT and also in FMRP-KO mice in the absence $(n=4, p<0.01)$ or in the presence $(n=4, p<0.01)$ of DHPG (Figure 4c). The activity of MAGL was conversely unchanged by DHPG in BC1-KOs ( $p>0.05$ compared with preDHPG), and was similar to that observed in FMRP-KO mice, both in basal condition and after stimulation with DHPG $(n=4, p>0.05)$ (Figure 4d). Notably, DHPG-induced increase of 2-AG contents did not reach statistical significance in $\mathrm{BC} 1-\mathrm{KO}$ mice $(n=4, p>0.05$ compared with pre-DHPG) suggesting, as in the case of FMRP-KO mice, that the metabolism of this endocannabinoid is also regulated by mechanisms independent of DAGL and MAGL activity (Di Marzo and Maccarrone, 2008) (Figure 4e). The potentiated neurophysiological effect of DHPG, however, indicates that the synaptic availability of $2-\mathrm{AG}$ is indeed enhanced by mGlu5R stimulation in BC1-KO mice.

As in FMRP-KO mice, AM251 was unable to increase per se mIPSC frequency in BC1-KO striatal neurons $(n=5 / 5$, $p>0.05$; not shown), also arguing against an endogenous endocannabinoid tone in these mice. Also, direct pharmacological stimulation of CB1Rs with HU210 produced similar inhibition of mIPSCs in WT $(n=6 / 8)$ and in BC1-KO striatal neurons $(n=7 / 8 ; p>0.05$ compared with HU210 effect in WT mice; Figure 4f). The inhibitory effect of HU210 on glutamate-mediated mEPSCs was also similar in WT $(n=5 / 5)$ and BC1-KO neurons $(n=5 / 5 ; p>0.05$; Figure $4 \mathrm{~g})$. 

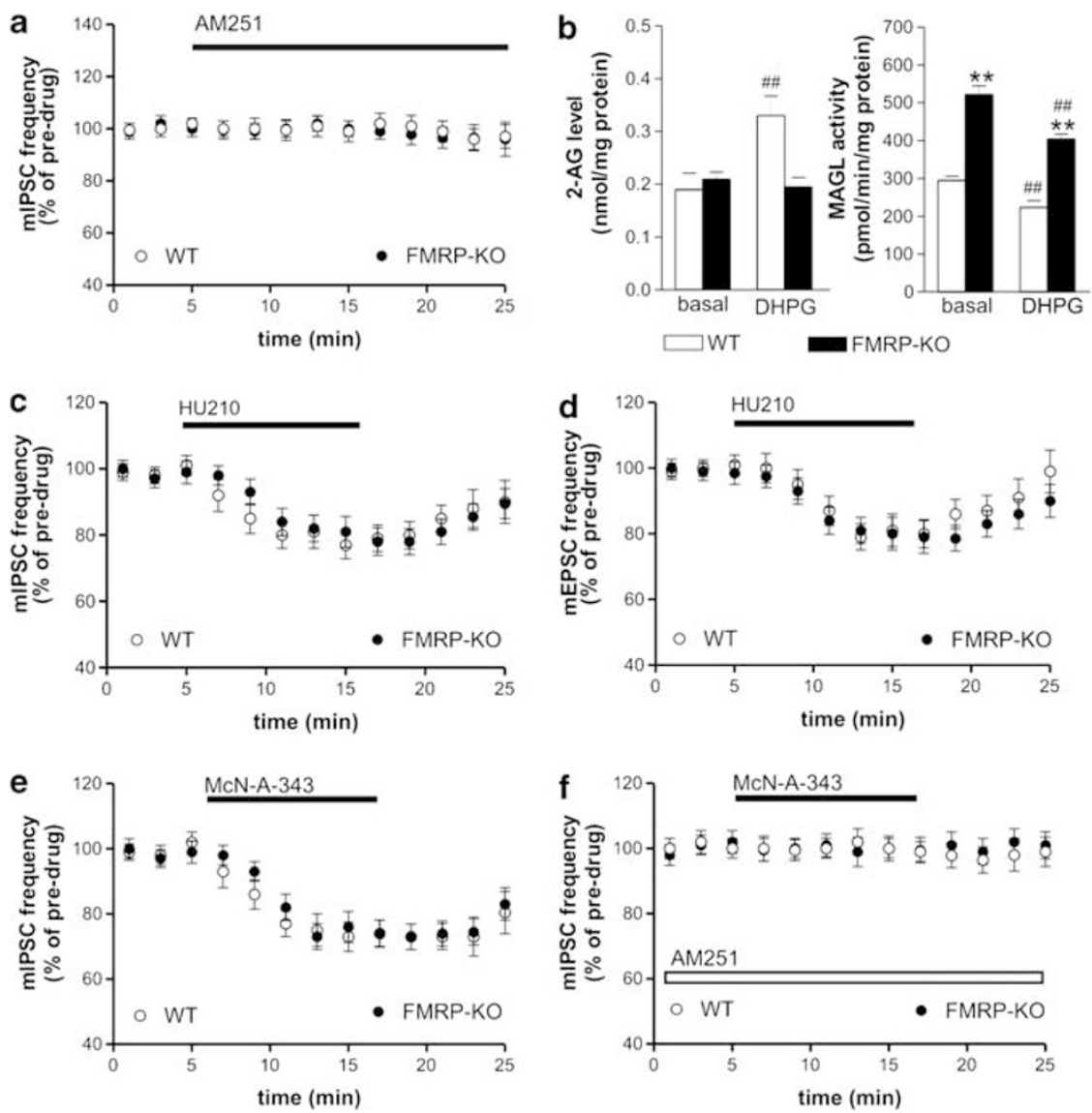

Figure 3 Effects of the direct and indirect MI-dependent modulation of CBIRs in FMRP-KO mice. (a) AM25I failed to increase mIPSC frequency in both WT and FMRP-KO neurons. (b) The histograms show that the genetic inactivation of fmrl remarkably increases MAGL activity in the absence (basal) or in the presence of DHPG (DHPG) without altering 2-AG levels. (c, d) The depressant effects of HU2 I 0 on mIPSC frequency (c) and on mEPSC frequency (d) were similar in WT and in FMRP-KO mice. (e) The reduction of mIPSC frequency induced by muscarinic MI receptors agonist McN-A-343, was similar in WT and in FMRP-KO mice. ( $f$ Preincubation with the $\mathrm{CBI}$ receptor antagonist AM25I prevented the depressant action of McN-A-343 in WT and in FMRP-KO mice. ${ }^{*} *$ $p<0.0$ I vs WT; ${ }^{\# \#} p<0.01$ vs pre-DHPG values.

\section{mGlu5/Endocannabinoid Interaction in Double FMRP-BC1-KO Mice}

The distinct mechanisms at the basis of FMRP- and $\mathrm{BC} 1$ RNA-mediated modulation of 2-AG metabolism suggests that the functional effects of $f m r l$ gene ablation might be attenuated in FMRP-KO mice by residual activity of $\mathrm{BC1}$ RNA and vice versa. According to this hypothesis, simultaneous ablation of FMRP and of BC1 RNA could exacerbate the synaptic defects seen in mice lacking FMRP or BC1 RNA only. We tested this hypothesis by measuring the effects of DHPG on striatal mIPSCs recorded from double FMRP-BC1-KO mice. In these double mutants, DHPG produced a dramatic inhibition of mIPSC frequency in all the recorded cells $(n=18 / 18, p<0.01)$, a result consistent with the idea that mGlu5R/endocannabinoid coupling is enhanced (Figure $5 a$ and $b$ ). Accordingly, both MPEP binding and DAGL activity were increased in FMRPBC1-KO mice $(n=4 p<0.01$ for each experimental group; Figure $5 \mathrm{c}$ ), as also observed in FMRP-KOs. Notably, both basal and DHPG-stimulated DAGL activity was higher in the double KOs (basal: $678 \pm 40 \mathrm{pmol} / \mathrm{min} / \mathrm{mg}$ protein; DHPG: $776 \pm 26 \mathrm{pmol} / \mathrm{min} / \mathrm{mg}$ protein) than in FMRP-KO (basal: $539 \pm 27 \mathrm{pmol} / \mathrm{min} / \mathrm{mg}$ protein; DHPG: $501 \pm 25 \mathrm{pmol} / \mathrm{min} /$ $\mathrm{mg}$ protein) and in BC1-KO mice (basal: $421 \pm 28 \mathrm{pmol} / \mathrm{min} /$ mg protein; DHPG: $657 \pm 28 \mathrm{pmol} / \mathrm{min} / \mathrm{mg}$ protein) $(p<0.05)(n=4$ for all conditions), while the activity of MAGL was comparable in the three KOs (FMRP-BC1-KO (basal): $522 \pm 23$, FMRP-KO (basal): $529 \pm 31, \mathrm{BC} 1-\mathrm{KO}$ (basal): $464 \pm 20 \mathrm{pmol} / \mathrm{min} / \mathrm{mg}$ protein; FMRP-BC1-KO (DHPG): $426 \pm 13$, FMRP-KO (DHPG): $417 \pm 27$, BC1-KO (DHPG): $404 \pm 27 \mathrm{pmol} / \mathrm{min} / \mathrm{mg}$ protein $)(p>0.05) \quad(n=4$ for all conditions; not shown). Together, these results suggest that 2-AG contents are higher in FMRP-BC1-KO mice than in FMRP-KOs and in BC1-KOs. In fact, direct determination of 2-AG revealed that the levels of this endocannabinoid, either in the absence or in the presence of DHPG, were significantly increased in the striatum of double mutants compared with WT, FMRP-KO, and BC1KO mice ( $n=4$ and $p<0.01$ for all conditions) (Figure $5 \mathrm{~d}$ ).

Striatal 2-AG levels in the double KOs were high enough to influence basal synaptic transmission in these mutants, because inhibition of CB1Rs with AM251 not only fully blocked the DHPG effects on mIPSCs $(n=8 / 8$, $p>0.05$; Figure 5e), but also increased per se mIPSC frequency $(n=8 / 8, p<0.05)$, as expected for a tonic activation of these receptors by endocannabinoids (Figure 5f).

As with FMRP-KO and BC1 RNA mice, HU210 inhibited mIPSC frequency $(n=8 / 10, p<0.01)$ to a similar extent in 

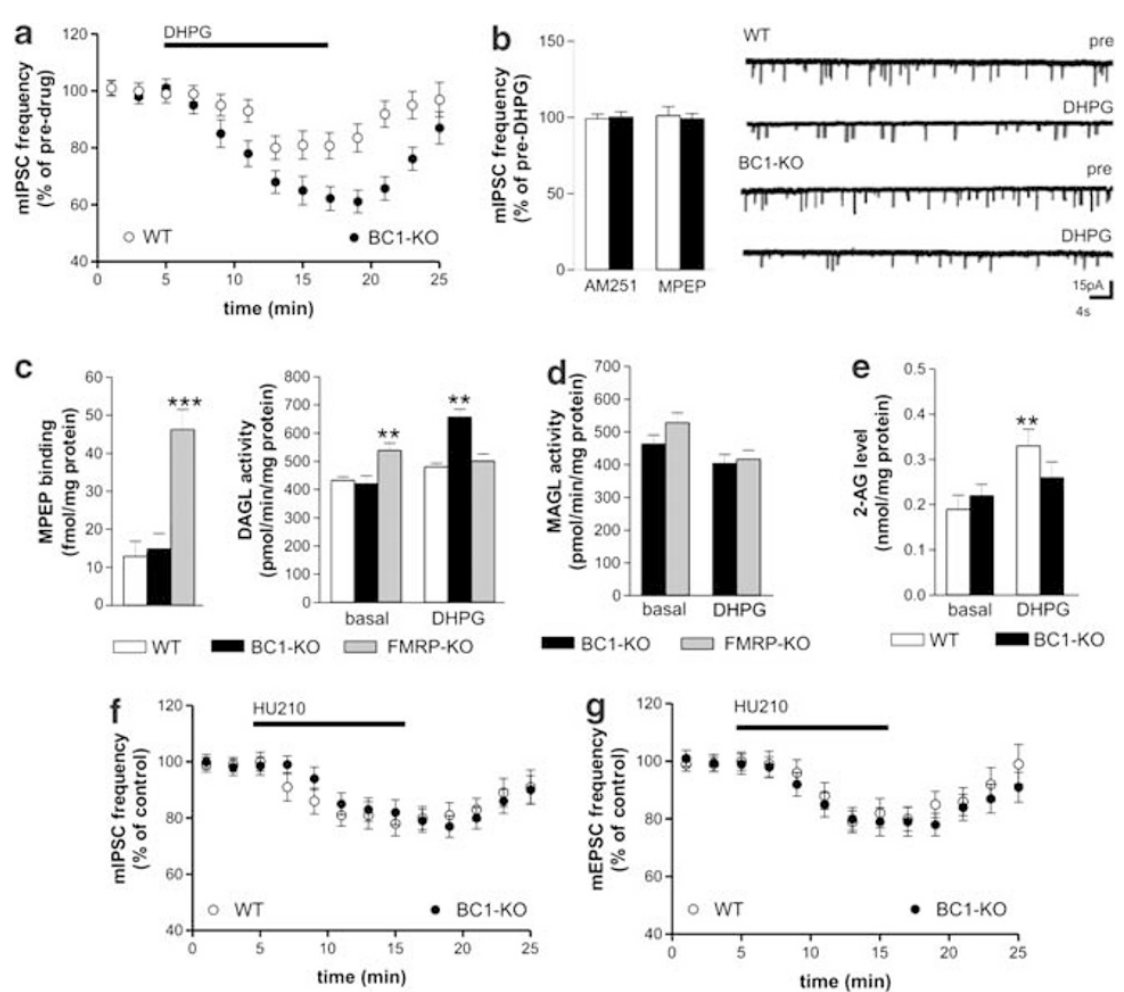

Figure $4 \mathrm{mGlu5R}$ /endocannabinoid interaction in $\mathrm{BCI}-\mathrm{KO}$ mice. $(\mathrm{a}, \mathrm{b})$ The reduction of mIPSC frequency after the application of DHPG was potentiated in $\mathrm{BCl}-\mathrm{KO}$ mice and was prevented (b) by preincubation with AM25I or with MPEP in WT and in $\mathrm{BCI}-\mathrm{KO}$ mice. The electrophysiological traces on the right are examples of voltage-clamp recordings before and during the application of DHPG in WT and in BCI-KO mice. (c) MPEP binding to mGlu5Rs and DAGL activity were similar in $\mathrm{BCI}-\mathrm{KO}$ and in WT mice in control condition (basal), and significantly reduced compared with FMRP-KO mice. The application of DHPG markedly increased DAGL activity in BCI-KO mice (DHPG). (d) The activity of MAGL was similar in BCI-KO and in FMRP-KO mice, both in basal condition and after stimulation with DHPG. (e) The graph shows that 2-AG levels were similar in WT and in BCI-KO mice in control condition (basal), whereas application of DHPG only increased 2-AG levels in WT mice (DHPG). (f, g) The depressant effects of HU2 I 0 on mIPSC frequency ( $f$ ) and on mEPSC frequency $(\mathrm{g})$ were similar in $\mathrm{WT}$ and in $\mathrm{BCI}-\mathrm{KO}$ mice. ${ }^{*} \mathrm{*} p<0.01$; **** $p<0.00 \mathrm{I}$.

FMRP-BC1-KO mice and in their respective control groups $(n=7 / 8, p>0.05$ compared with the synaptic currents recorded in mutants), indicating that the activity of CB1Rs controlling striatal GABA transmission was normal following isolated or combined ablation of FMRP and BC1 RNA (Figure 5g).

\section{DISCUSSION}

A bulk of data has already proposed a strong association between altered FMRP activity and abnormal signaling mediated by mGluRs. Accordingly, stimulation of mGluRs by DHPG has been shown to induce FMRP translation (Weiler et al, 1997) and translocation at dendritic spines (Ferrari et al, 2007), raising the possibility that FMRP mediates some of the synaptic effects of mGluR activation. In line with this hypothesis, mGlu5R-mediated long-term potentiation (LTP) is absent in the neocortex of FMRP-KO mice (Wilson and Cox, 2007). On the other hand, FMRP and mGlu5R oppose to each other in regulating hippocampal synaptic plasticity, because the absence of FMRP has been convincingly associated with normal LTP but increased mGlu5R-dependent long-term depression (LTD) in this brain area (Huber et al, 2002; Bear et al, 2004). Based on these findings, it can be concluded that both positive and negative interactions exist between FMRP and mGlu5Rs, and that one type of interaction emerges over the other depending on the specific paradigm of synaptic plasticity explored and the specific neuronal system under investigation.

To date, only LTP and LTD phenomena have been studied to address mGluR function in FXS models, although these receptors mediate other important roles in the brain. Exploring mGluR function in other contexts than synaptic plasticity and in other brain areas than the hippocampus and the cerebral cortex is therefore essential to confirm the idea that FXS symptoms result from a widespread dysregulation of mGlu5R activity (Dölen et al, 2007; Dölen and Bear, 2008).

In this study, we provided evidence that mGlu5Rdependent endocannabinoid activity and metabolism is altered in striatal neurons lacking FMRP. The results of this study indicate that mGlu5R-driven endocannabinoid signaling in the striatum is under the control of both FMRP and $\mathrm{BC} 1 \mathrm{RNA}$, critical translational repressors in neurons and at central synapses (Bagni and Greenough, 2005; Bassell and Warren, 2008, Zukin et al, 2009). Some physiological effects of mGlu5R stimulation have already been reported to follow rapid synaptic protein synthesis (Huber et al, 2000; Bear et al, 2004; Shin et al, 2004). DHPG-stimulated protein synthesis is regulated by both BC1 RNA (Zhong et al, 2009) and FMRP (Bear et al, 2004; Ferrari et al, 2007; Dölen and Bear, 2008; Napoli et al, 2008) through complex and only 


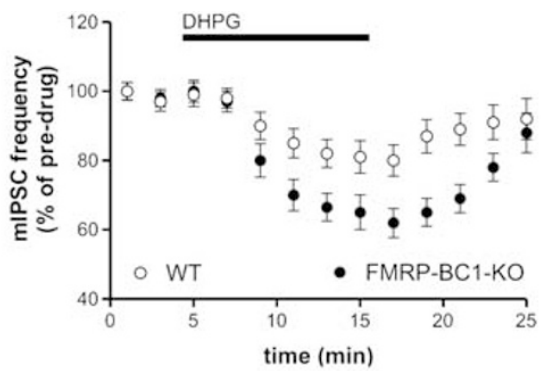

$\mathbf{b}_{W T}$
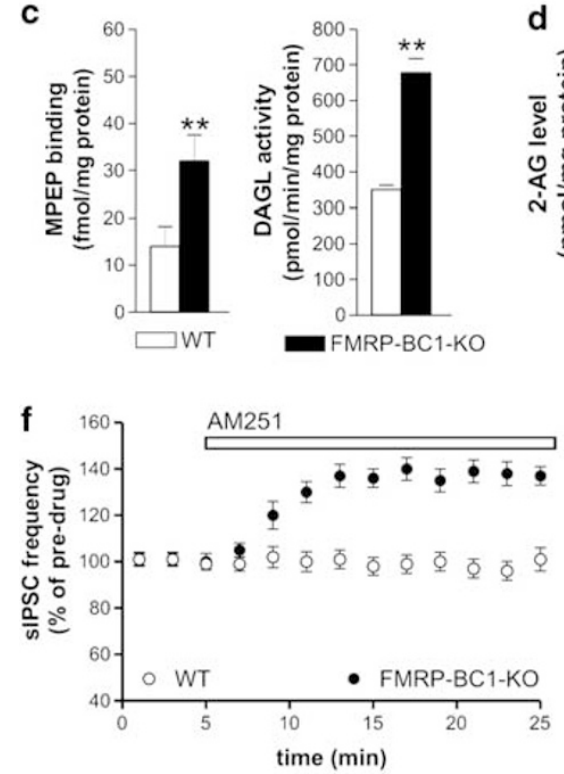
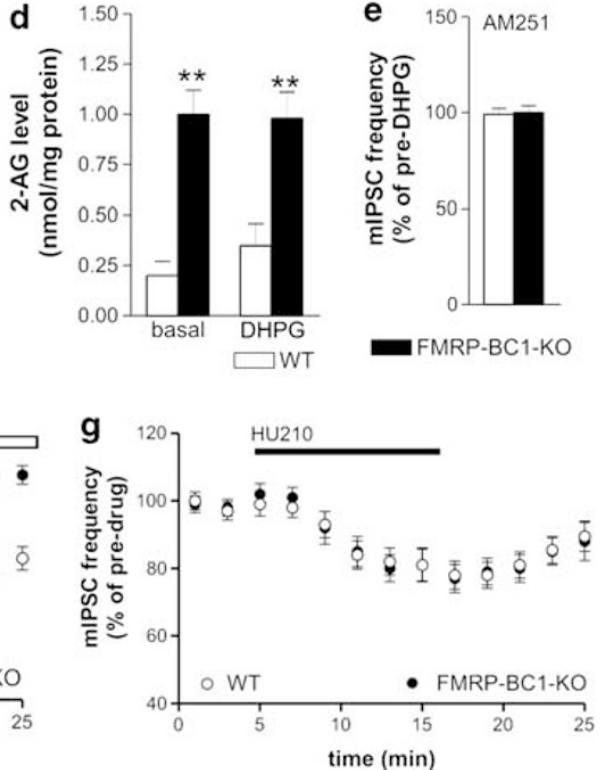

Figure $5 \mathrm{mGlu5R/endocannabinoid} \mathrm{interaction} \mathrm{in} \mathrm{double} \mathrm{FMRP-BCI-KO} \mathrm{mice.} \mathrm{(a)} \mathrm{The} \mathrm{reduction} \mathrm{of} \mathrm{mIPSC} \mathrm{frequency} \mathrm{after} \mathrm{the} \mathrm{application} \mathrm{of} \mathrm{DHPG} \mathrm{was}$ potentiated in FMRP-BCI-KO mice. (b) The electrophysiological traces are examples of voltage-clamp recordings before and during the application of DHPG in WT and FMRP-BCI-KO mice. (c) Simultaneous ablation of FMRP and of BCI RNA markedly increased MPEP binding to mGlu5Rs and DAGL activity in the striatum. (d) The histogram shows that 2-AG levels were remarkably increased in FMRP-BCI-KO mice both in the absence (basal) and in the presence of DHPG stimulation (DHPG). (e) Preincubation with AM25I prevented the depressant action of DHPG in WT and FMRP-BCI-KO mice. (f) AM25I increased per se mIPSC frequency in FMRP-BCI-KO mice. (g) The depressant effects of HU2 IO on mIPSC frequency were similar in WT and in FMRP-BCl-KO mice. ${ }^{*} *$ $p<0.01$.

partially elucidated mechanisms. Accordingly, BC1 RNA acts as a physiological constraint in the mGlu5R-driven stimulation of PSD-95 but also of FMRP synthesis (Zhong et al, 2009). On the other hand, the two translational repressors BC1 RNA and FMRP can cooperate in some instances, either by physical interaction (Zalfa et al, 2003; Johnson et al, 2006; Centonze et al, 2008) or by coordinate, although non-identical, actions on a common molecular pathway (present work).

Our data are in line with previous findings indicating that both FMRP and BC1 RNA function as repressive counterbalance to DHPG-stimulated effects, because ablation of both molecules has been associated with increased mGlu5R signaling (Dölen et al, 2007; Dölen and Bear, 2008; Zhong et al, 2009). Here, we have shown that a crucial effect of mGlu5R activation, namely the stimulation of 2-AGmediated endocannabinoid signaling, is under the control of FMRP and of BC1 RNA. Both FMRP and BC1 RNA ablation, in fact, enhanced the activity of DAGL, the main responsible enzyme for 2-AG synthesis (Maccarrone et al, 2008). Although these data indicate that mGlu5R/DAGL coupling is reduced under physiological conditions by the activity of the two translational repressors, our data also indicate that the mechanisms at the basis of FMRP- and BC1 RNA-dependent endocannabinoid regulation are remarkably distinct. Accordingly, DAGL activity was potentiated in BC1-KO mice only after DHPG application, whereas in FMRP-animals DAGL activity was higher both in basal conditions and after stimulation with DHPG. The two mutants also differed significantly in terms of mGlu5R expression, which was dramatically elevated only in FMRPKO mice. Together, these data might indicate that FMRP controls mGlu5R/2-AG coupling by regulating protein expression of mGlu5Rs, whereas $\mathrm{BC1}$ RNA regulates a downstream step in this interaction. Notably the defects of endocannabinoid signaling seen in isolated FMRP- and $\mathrm{BC} 1-\mathrm{KOs}$ were dramatically exacerbated by combined FMRP- and BC1 RNA-genetic disruption, as expected for a non-identical action of the two repressors on the chain of events responsible for mGlu5R/2-AG coupling.

The overexpression of mGlu5Rs only in FMRP lacking mice might also contribute to explain why DAGL was overactive in these KOs also in basal condition. Even in the absence of DHPG, in fact, mGlu5Rs are likely activated in 
FMRP-KO mice by endogenous glutamate, whose action is conversely undetectable in WT and $\mathrm{BC} 1-\mathrm{KO}$ animals with lower receptor expression.

In FMRP-KO mice, however, a remarkable proportion of striatal neurons failed to respond to DHPG, whereas in both FMRP- and BC1-KO mice, the direct activation of CB1Rs with HU210 evoked normal IPSC suppression in almost the totality of the recorded neurons. Together, these data suggest that FMRP, but not BC1 RNA genetic ablation, also disrupts the activity of a certain population of mGluRs. The reason of the heterogeneous sensitivity to DHPG of FMRPKO striatal neurons is unknown, but it can be postulated that it reflects the heterogeneity of striatopallidal and striatonigral projection cells in terms of preferential form of synaptic plasticity expressed. According to the observation that LTP- (Wilson and Cox, 2007) and LTD-preferring (Huber et al, 2002) neurons respond to FMRP ablation by reducing or enhancing, respectively, their sensitivity to mGlu5R activation, it can be hypothesized that FMRP-KO striatal neurons with enhanced response to DHPG are LTDpreferring striatopallidal cells, whereas those with reduced response to this agonist are striatonigral neurons, which are more prone to LTP induction (Surmeier et al, 2007). At this stage, however, this interpretation is merely speculative, and needs to be supported by further data.

The striatum is a brain area involved in FXS (Menon et al, 2004), it expresses high levels of markers of the endocannabinoid system (Maccarrone et al, 2008), and is heavily regulated by glutamate acting through both ionotropic and mGluRs (Ding et al, 2008). Although primarily involved in motor control, in fact, the striatum has important roles in cognitive and motivational functions, explaining why abnormal striatal activity has been proposed at the basis of stereotypies, hyperactivity, and perseverative motor behavior frequently observed in FXS patients (Freund and Reiss, 1991; Hagerman, 2006). Brain imaging studies have identified a close association between reduced expression of FMRP and morphological and functional abnormalities of the basal ganglia in FXS patients. Accordingly, FXS patients present frontostriatal deficits and larger striatum than normal, abnormalities that tightly correlate with the severity of the disease (Menon et al, 2004). This evidence suggests that altered glutamate-mediated corticostriatal transmission has a major role in FXS symptoms, but so far only one study has addressed the physiological properties of glutamatemediated transmission in the striatum of FMRP-KO mice. That study reported that excitatory transmission mediated by ionotropic glutamate receptors was unaltered in the striatum of FXS mice (Centonze et al, 2008).

In conclusion, this is the first study addressing endocannabinoid system in a model of FXS. Our results show that dysfunctional mGlu5R signaling leads to abnormal 2-AG metabolism and physiological activity, and indicate that inhibition of 2-AG synthesis or activity at CB1Rs might be a useful treatment option in FXS patients. In this respect, recent investigations suggest that this modulation could be achieved not only by direct pharmacological blockade of CB1Rs, but also indirectly, for example through the inhibition of anandamide degradation or the stimulation of transient receptor potential vanilloid 1 (TRPV1) channels. These two components of the endocannabinoid system, in fact, have been shown to selectively interact with
mGlu5R/2-AG coupling in striatal neurons (Di Marzo and Maccarrone, 2008; Maccarrone et al, 2008), and might interfere with the synaptic alterations seen after FMRP ablation with less side effects than those of widespread pharmacological inhibition of CB1Rs, which control not only GABA but also glutamate synapses.

\section{ACKNOWLEDGEMENTS}

This investigation was supported by the Italian National Ministero dell'Università e Ricerca to $\mathrm{MM}$ and to $\mathrm{DC}$, by the Italian National Ministero della Salute to DC, by Fondazione TERCAS (Research Program 2009-2012) to MM, and by the FIRB international, VIB and FWO to CB. We thank Valentina Mercaldo for some preliminary data.

\section{DISCLOSURE}

The authors declare no conflict of interest.

\section{REFERENCES}

Bagni C, Greenough WT (2005). From mRNP trafficking to spine dysmorphogenesis: the roots of fragile $\mathrm{X}$ syndrome. Nat Rev Neurosci 6: 376-387.

Bassell GJ, Warren ST (2008). Fragile X syndrome: loss of local mRNA regulation alters synaptic development and function. Neuron 60: 201-214.

Bear MF, Huber KM, Warren ST (2004). The mGluR theory of fragile X mental retardation. Trends Neurosci 27: 370-377.

Bisogno T, Cascio MG, Saha B, Mahadevan A, Urbani P, Minassi A et al (2006). Development of the first potent and specific inhibitors of endocannabinoid biosynthesis. Biochim Biophys Acta 1761: 205-212.

Bisogno T, Howell F, Williams G, Minassi A, Cascio MG, Ligresti A et al (2003). Cloning of the first sn1-DAG lipases points to the spatial and temporal regulation of endocannabinoid knockout in the brain. J Cell Biol 163: 463-468.

Bonsi P, Platania P, Martella G, Madeo G, Vita D, Tassone A et al (2008). Distinct roles of group I mGlu receptors in striatal function. Neuropharmacology 55: 392-395.

Centonze D, Rossi S, Mercaldo V, Napoli I, Ciotti MT, De Chiara V et al (2008). Abnormal striatal GABA transmission in the mouse model for the fragile X syndrome. Biol Psychiatry 63: 963-973.

Centonze D, Rossi S, Napoli I, Mercaldo V, Lacoux C, Ferrari F et al (2007a). The brain cytoplasmic RNA $\mathrm{BC} 1$ regulates dopamine D2 receptor-mediated transmission in the striatum. J Neurosci 27: 8885-8892.

Centonze D, Rossi S, Prosperetti C, Gasperi V, De Chiara V, Bari M et al (2007b). Endocannabinoids limit metabotropic glutamate 5 receptor-mediated synaptic inhibition of striatal principal neurons. Mol Cell Neurosci 35: 302-310.

Di Marzo V, Maccarrone M (2008). FAAH and anandamide: is 2-AG really the odd one out? Trends Pharmacol Sci 29: 229-233.

Ding J, Peterson JD, Surmeier DJ (2008). Corticostriatal and thalamostriatal synapses have distinctive properties. J Neurosci 28: 6483-6492.

Dinh TP, Carpenter D, Leslie FM, Freund TF, Katona I, Sensi SL et al (2002). Brain monoglyceride lipase participating in endocannabinoid inactivation. Proc Natl Acad Sci USA 99: 10819-10824.

Dölen G, Bear MF (2008). Role for metabotropic glutamate receptor 5 (mGluR5) in the pathogenesis of fragile $\mathrm{X}$ syndrome. J Physiol 586: 1503-1508. 
Dölen G, Osterweil E, Rao BS, Smith GB, Auerbach BD, Chattarji S et al (2007). Correction of fragile $\mathrm{X}$ syndrome in mice. Neuron 56: $955-962$.

Domenici MR, Potenza RL, Martire A, Coccurello R, Pèzzola A, Reggio $\mathrm{R}$ et al (2005). Chronic treatment with the mGlu5R antagonist MPEP reduces the functional effects of the mGlu5R agonist CHPG in the striatum of 6-hydroxydopamine-lesioned rats: possible relevance to the effects of mGlu5R blockade in Parkinson's disease. J Neurosci Res 80: 646-654.

Ferrari F, Mercaldo V, Piccoli G, Sala C, Cannata S, Achsel T et al (2007). The fragile $\mathrm{X}$ mental retardation protein-RNP granules show an mGluR-dependent localization in the post-synaptic spines. Mol Cell Neurosci 34: 343-354.

Freund LS, Reiss AL (1991). Cognitive profiles associated with the fra $(\mathrm{X})$ syndrome in males and females. Am J Med Genet 38: 542-547.

Giuffrida A, Rodriguez de Fonseca F, Nava F, Loubet-Lescoulié P, Piomelli D (2000). Elevated circulating levels of anandamide after administration of the transport inhibitor, AM404. Eur J Pharmacol 408: 161-168.

Hagerman RJ (2006). Lessons from fragile X regarding neurobiology, autism, and neurodegeneration. J Dev Behav Pediatr 27: 63-74.

Hoover HS, Blankman JL, Niessen S, Cravatt BF (2008). Selectivity of inhibitors of endocannabinoid biosynthesis evaluated by activity-based protein profiling. Bioorg Med Chem Lett 18: 5838-5841.

Huber KM, Gallagher SM, Warren ST, Bear MF (2002). Altered synaptic plasticity in a mouse model of fragile $\mathrm{X}$ mental retardation. Proc Natl Acad Sci USA 99: 7746-7750.

Huber KM, Kayser MS, Bear MF (2000). Role for rapid dendritic protein synthesis in hippocampal mGluR-dependent long-term depression. Science 288: 1254-1257.

Johnson EM, Kinoshita Y, Weinreb DB, Wortman MJ, Simon R, Khalili $\mathrm{K}$ et al (2006). Role of Pur alpha in targeting mRNA to sites of translation in hippocampal neuronal dendrites. J Neurosci Res 83: 929-943.

Jung KM, Mangieri R, Stapleton C, Kim J, Fegley D, Wallace M et al (2005). Stimulation of endocannabinoid formation in brain slice cultures through activation of group I metabotropic glutamate receptors. Mol Pharmacol 68: 1196-1202.

Maccarrone M, De Chiara V, Gasperi V, Viscomi MT, Rossi S, Oddi $S$ et al (2009). Lipid rafts regulate 2-arachidonoylglycerol metabolism and physiological activity in the striatum. J Neurochem 109: 371-381.

Maccarrone M, Rossi S, Bari M, De Chiara V, Fezza F, Musella A et al (2008). Anandamide inhibits metabolism and physiological actions of 2-arachidonoylglycerol in the striatum. Nat Neurosci 11: $152-159$.
Menon V, Leroux J, White CD, Reiss AL (2004). Frontostriatal deficits in fragile $\mathrm{X}$ syndrome: relation to FMR1 gene expression. Proc Natl Acad Sci USA 101: 3615-3620.

Napoli I, Mercaldo V, Pilo Boyl P, Eleuteri B, Zalfa F, De Rubeis S et al (2008). The fragile $X$ mental retardation protein represses activity-dependent translation through CYFIP1, a new 4E-BP'. Cell 134: 1042-1054.

Narushima M, Uchigashima M, Fukaya M, Matsui M, Manabe T, Hashimoto $\mathrm{K}$ et al (2007). Tonic enhancement of endocannabinoid-mediated retrograde suppression of inhibition by cholinergic interneuron activity in the striatum. J Neurosci 27: 496-506.

Schuman EM, Dynes JL, Steward O (2006). Synaptic regulation of translation of dendritic mRNAs. J Neurosci 26: 7143-7146.

Shin CY, Kundel M, Wells DG (2004). Rapid, activity-induced increase in tissue plasminogen activator is mediated by metabotropic glutamate receptor-dependent mRNA translation. J Neurosci 24: 9425-9433.

Surmeier DJ, Ding J, Day M, Wang Z, Shen W (2007). D1 and D2 dopamine-receptor modulation of striatal glutamatergic signaling in striatal medium spiny neurons. Trends Neurosci 30: 228-235.

Sutton MA, Schuman EM (2006). Dendritic protein synthesis, synaptic plasticity, and memory. Cell 1: 49-58.

Wang H, Iacoangeli A, Lin D, Williams K, Denman RB, Hellen CU et al (2005). Dendritic BC1 RNA in translational control mechanisms. J Cell Biol 171: 811-821.

Wang $\mathrm{H}$, Iacoangeli A, Popp S, Muslimov IA, Imataka $\mathrm{H}$, Sonenberg $\mathrm{N}$ et al (2002). Dendritic BC1 RNA: functional role in regulation of translation initiation. J Neurosci 22: 10232-10241.

Weiler IJ, Irwin SA, Klintsova AY, Spencer CM, Brazelton AD, Miyashiro K et al (1997). Fragile X mental retardation protein is translated near synapses in response to neurotransmitter activation. Proc Natl Acad Sci USA 94: 5395-5400.

Wilson BM, Cox CL (2007). Absence of metabotropic glutamate receptor-mediated plasticity in the neocortex of fragile $\mathrm{X}$ mice. Proc Natl Acad Sci USA 104: 2454-2459.

Zalfa F, Achsel T, Bagni C (2006). mRNPs, polysomes or granules: FMRP in neuronal protein synthesis. Curr Opin Neurobiol 16: 265-269.

Zalfa F, Giorgi M, Primerano B, Moro A, Di Penta A, Reis S et al (2003). The fragile $\mathrm{X}$ syndrome protein FMRP associates with BC1 RNA and regulates the translation of specific mRNAs at synapses. Cell 112: 317-327.

Zhong J, Chuang SC, Bianchi R, Zhao W, Lee H, Fenton AA et al (2009). BC1 regulation of metabotropic glutamate receptormediated neuronal excitability. J Neurosci 29: 9977-9986.

Zukin R, Richter JD, Bagni C (2009). Signals, synapses, and synthesis: how new proteins control plasticity. Front Neural Circuits 3: 14, doi:10.3389/neuro.04.014.2009. 